\title{
Unconventional collective normal-mode coupling in quantum-dot-based bimodal microlasers
}

\author{
M. Khanbekyan, ${ }^{1, *}$ H. A. M. Leymann, ${ }^{2}$ C. Hopfmann, ${ }^{1}$ A. Foerster, ${ }^{2}$ C. Schneider, ${ }^{3}$ S. Höfling,,${ }^{3, \dagger}$ \\ M. Kamp, ${ }^{3}$ J. Wiersig, ${ }^{2}$ and S. Reitzenstein ${ }^{1}$ \\ ${ }^{1}$ Institut für Festkörperphysik, Technische Universität Berlin, Hardenbergstraße 36, D-10623 Berlin, Germany \\ ${ }^{2}$ Institut für Theoretische Physik, Otto-von-Guericke-Universität Magdeburg, Postfach 4120, D-39016 Magdeburg, Germany \\ ${ }^{3}$ Technische Physik and Wilhelm Conrad Röntgen Research Center for Complex Material Systems, Physikalisches Institut, Universität \\ Würzburg, Am Hubland, D-97074 Würzburg, Germany
}

(Received 13 July 2014; published 28 April 2015; publisher error corrected 20 May 2015)

\begin{abstract}
We analyze the occurrence of normal-mode coupling (NMC) in bimodal lasers attributed to the collective interaction of the cavity field with a mesoscopic number of quantum dots (QDs). In contrast to the conventional $\mathrm{NMC}$, here we observe locking of the frequencies and splitting of the linewidths of the system's eigenmodes in the coherent coupling regime. The theoretical analysis of the incoherent regime is supported by experimental observations where the emission spectrum of one of the orthogonally polarized modes of a bimodal QD micropillar laser demonstrates a distinct two-peak structure.
\end{abstract}

DOI: 10.1103/PhysRevA.91.043840

PACS number(s): 42.55.Sa, 42.50.Ar, 42.50.Ct

Introduction. The study of cavity quantum electrodynamics (CQED) in the strong-coupling regime between atomlike emitters and the confined light field of microcavities has been a subject of considerable attention. In the traditional CQED, low-mode volume resonators are used to enhance the coupling rate $g$ between a single emitter and the field in comparison to the system damping rates. Prominent realizations of the strong coupling include experimental demonstrations of reversible exchange of excitation between a single emitter and the field from both atomic [1-3] and solid-state [4,5] systems. Typical evidence of the strong-coupling regime represents splitting of the two degenerate modes, i.e., normal-mode splitting, which is a consequence of normal-mode coupling (NMC), e.g., between the emitter polarization mode and the field mode leading to a doublet cavity transmission spectrum [6]. In addition, NMC occurs in exciton-photon and phonon-photon interactions [7] and optomechanical phenomena [8], where the cavity field couples to a mechanical mode [9].

In view of the variety of implications of the regime of coherent coupling (see, e.g., [10]), a different approach to achieve strong coupling has also attracted much attention. Instead of reducing the cavity-mode volume to achieve large $g$, the number of emitters $N$ interacting with the field can be increased, leading to the collective strong-coupling regime, where the coupling rate scales as $\sqrt{N} g[11,12]$. Various experimental observations of cavity-mode spectra proportional to $\sqrt{N} g$ due to the collective coherent coupling with two $[13,14]$ or multiple $[15,16]$ emitters have been made, including the case of a multimode cavity [17]. In solid-state systems, the coherent coupling between a cavity mode and an ensemble of emitters has been achieved in the classical regime with semiconductor quantum wells $[7,18]$. However, in the quantum regime the significant inhomogeneous broadening of emission from self-assembled quantum dots (QDs) has so far hindered the observation of collective coherent coupling for semiconductor-based quantum emitters.

\footnotetext{
*mikayel.khanbekyan@ovgu.de

${ }^{\dagger}$ Present address: SUPA, School of Physics and Astronomy, University of St. Andrews, St. Andrews KY16 9SS, United Kingdom.
}

In many different situations (see, e.g., Refs. [1-7,7-18]), by convention coherent coupling of two (nearly degenerate) modes is commonly explained by studying the eigenvalues of the system,

$$
\begin{aligned}
\Lambda_{ \pm}= & \frac{\omega_{1}+\omega_{2}}{2}-i \frac{\gamma_{1}+\gamma_{2}}{4} \\
& \pm\left\{\left[\left(\omega_{1}-\omega_{2}\right) / 2-i\left(\gamma_{1}-\gamma_{2}\right) / 4\right]^{2}+N g^{2}\right\}^{1 / 2}
\end{aligned}
$$

where $\omega_{1,2}$ and $\gamma_{1,2}$ are the frequencies and decay rates of the modes, respectively. Analysis of this expression reveals that in the resonant case $\left(\omega_{1}=\omega_{2}\right)$ for $\sqrt{N} g<\left|\gamma_{1}-\gamma_{2}\right| / 4$ the square-root term is fully imaginary and modifies the decay rates of the modes. Further, for $\sqrt{N} g>\left|\gamma_{1}-\gamma_{2}\right| / 4$ it becomes real and the frequencies exhibit a splitting attributed to NMC.

Coherent coupling is also relevant to laser physics for achieving the regime of bistable lasing of two-mode lasers $[19,20]$. In particular, in the case of large pump rates when the strong-coupling regime of the emitter-field interaction is achieved and the Rabi frequency is larger than the mode separation, mode locking has been observed [21,22]. Otherwise, bimodal cavities are investigated in the context of single-photon generation with whispering-gallery-mode resonators $[23,24]$, where an atom strongly interacts with two cavity modes.

Here, we show that interaction of the modes of a passive bimodal microcavity (cavity modes) with a mesoscopic number of quantum emitters induces unconventional coherent coupling between these modes in the lasing regime. In contrast to the conventional NMC described above, here, in the case of near-resonant cavity modes the eigenmodes of the total active system (eigenmodes) exhibit frequency locking, and the effective coupling rate with the emitters $\sqrt{N} g$ induces splitting of the linewidths of the eigenmodes. Further, for sufficiently large spectral splitting between the cavity modes, the incoherent coupling between the modes leads to a mixing of the "bare" cavity-mode frequencies in the emission spectrum. We report below the experimental observation of this mode mixing for bimodal micropillar lasers. Moreover, our theoretical study shows that in the case of incoherent coupling and approximately equal mode-QD coupling rates, 
the eigenmode linewidths demonstrate locking, leading to almost equal values of the coherence times of the cavity-mode emission. This intriguing and unexpected scenario has been recently observed for bimodal microlasers [25], where the inferior mode which exhibits large superthermal intensity fluctuations indeed has a coherence time of the same order of magnitude as the dominant lasing mode.

Theoretical model. We consider a system consisting of two orthogonal cavity modes with the Hamiltonian $H_{\mathrm{ph}}=$ $\sum_{\xi} \hbar \omega_{\xi} b_{\xi}^{\dagger} b_{\xi}\left(\xi=1,2 ; b_{\xi}\right.$ is the annihilation operator of the photon in the $\xi$ th cavity mode) and QDs as gain medium, where the $s$-shell transition is resonantly coupled to the cavity electromagnetic field and the $p$-shell is pumped at a constant rate. The QD part of the Hamiltonian consists of single-particle contributions for conduction- and valence-band carriers with the energies $\varepsilon_{j}^{c, v}, H_{\text {carr }}^{0}=\sum_{j}\left(\varepsilon_{j}^{c} c_{j}^{\dagger} c_{j}+\varepsilon_{j}^{v} v_{j}^{\dagger} v_{j}\right)$, and the two-particle Coulomb interaction (see [25]). For the total Hamiltonian of the system the free Hamiltonian terms are complemented with the interaction energy of the QDs with the electromagnetic field; the latter in the dipole approximation reads

$$
H_{\mathrm{D}}=-i \sum_{\xi, j} g_{\xi j}\left(c_{j}^{\dagger} v_{j}+v_{j}^{\dagger} c_{j}\right) b_{\xi}+\text { H.c. }
$$

where the approximation of equal wave-function envelopes for conduction- and valence-band states is used and the coupling strengths $g_{\xi j}$ are assumed to be positive and real.

The coherent features of the output radiation are described by the (normalized) first-order correlation function

$$
g_{\xi}^{(1)}(t, \tau)=\frac{G_{\xi \xi}^{(1)}(t, \tau)}{\left\langle b_{\xi}^{\dagger}(t) b_{\xi}(t)\right\rangle}, \quad \xi=1,2,
$$

with $G_{\xi \xi^{\prime}}^{(1)}(t, \tau)=\left\langle b_{\xi}^{\dagger}(t+\tau) b_{\xi^{\prime}}(t)\right\rangle$. The coherence times and the frequency spectra are given, respectively, by

$$
\begin{gathered}
\tau_{\xi}^{c}=2 \int_{0}^{\infty} d \tau\left|g_{\xi}^{(1)}(\tau)\right|^{2}, \\
S_{\xi}(\omega)=2 \operatorname{Re} \int_{0}^{\infty} d \tau g_{\xi}^{(1)}(\tau) e^{-2 \pi i \tau \omega} .
\end{gathered}
$$

We restrict ourselves to the case of continuous-wave excitation and assume that two-time quantities such as the correlation function $g_{\xi}^{(1)}(t, \tau)$ are $t$-time independent in the steady-state regime for large enough times $t$. Therefore, the two-time evolution problem can be separated into two single-time problems, which is analogous to the method of the quantum regression theorem [26]. Then the equations of motion with respect to the delay time $\tau$ can be solved with initial values given by the stationary steady-state result of the $t$-time problem [27]. The Heisenberg equations of motion for expectation values of the quantities of interest lead to a hierarchy problem which can be treated by the cluster expansion (see $[25,28]$ ).

Further, assuming carrier generation in the $p$-shell at a fixed rate we derive a system of Heisenberg equations of motion and introduce phenomenological dissipative terms, where both pump and dissipative processes are described by a Lindblad form [29]. To obtain the dynamical equations of first-order coherence the cluster expansion up to doublet level is required, which implies the following factorization [in the following we omit the dependence on the time $t$, as $b_{\xi}(\tau) \equiv b_{\xi}(t, \tau)$, $b_{\xi} \equiv b_{\xi}(t, 0)$, etc.]

$$
\left\langle c_{j}^{\dagger}(\tau) c_{j}(\tau) b_{\xi}^{\dagger} b_{\xi}(\tau)\right\rangle \approx\left\langle c_{j}^{\dagger}(\tau) c_{j}(\tau)\right\rangle\left\langle b_{\xi}^{\dagger} b_{\xi}(\tau)\right\rangle,
$$

where $\left\langle c_{j}^{\dagger}(\tau) c_{j}(\tau)\right\rangle$ in the stationary regime is $\tau$-time independent. Thus, assuming identical QDs with equal transition energies and coupling rates $g_{\xi} \equiv g_{\xi j}$, we obtain the closed system of linear differential equations for the correlation functions $G_{\xi \zeta} \equiv G_{\xi \zeta}^{(1)}(\tau)$ and $P_{\xi}(\tau) \equiv P_{\xi j}(\tau)=\left\langle c_{j}^{\dagger}(\tau) v_{j}(\tau) b_{\xi}\right\rangle$ in the rotating-wave approximation and in the frame rotating at $\varepsilon_{j}^{c}-\varepsilon_{j}^{v}$,

$$
\begin{aligned}
& \frac{d}{d \tau} P_{\xi}(\tau)=-\Gamma P_{\xi}(\tau)+I g_{1} G_{1 \xi}(\tau)+I g_{2} G_{2 \xi}(\tau), \\
& \frac{d}{d \tau} G_{1 \xi}(\tau)=\left(i \Delta_{1}-\frac{1}{2} \kappa_{1}\right) G_{1 \xi}(\tau)+N g_{1} P_{\xi}(\tau), \\
& \frac{d}{d \tau} G_{2 \xi}(\tau)=\left(i \Delta_{2}-\frac{1}{2} \kappa_{2}\right) G_{2 \xi}(\tau)+N g_{2} P_{\xi}(\tau),
\end{aligned}
$$

where $\Delta_{\xi} \equiv \Delta_{\xi j}=\varepsilon_{j}^{c}-\varepsilon_{j}^{v}-\hbar \omega_{\xi}$ is the detuning of the cavity modes from the QD transition, and $\kappa_{1,2}$ describe cavitymode losses. The inhomogeneous broadening is approximated by the spectral line broadening rate $\Gamma$ in Eq. (6). The excitation of emitters with a given pump rate is encoded into the steadystate inversion per QD, $I \equiv I_{j}(t)=\left\langle c_{j}^{\dagger} c_{j}\right\rangle-\left\langle v_{j}^{\dagger} v_{j}\right\rangle$, which represents an important pump-rate-dependent parameter for the $\tau$ dynamics.

Normal-mode coupling. The system of six equations above consists of two independent subsystems with $\xi=1,2$. Autocorrelation functions of two cavity modes $G_{\xi \xi}^{(1)}(\tau)$ are coupled to each other indirectly, namely, through coupling to $P_{\xi}(\tau)$, representing the common gain medium. To provide a simple understanding of the coupling of the two cavity modes, we use an approximation of fast relaxation of $P_{\xi}(\tau)$ compared to the time scale of the dynamics of $G_{\xi \zeta}(\tau)$-typically valid for semiconductor systems [30]. Then we formally insert $(d / d \tau) P_{\xi}(\tau)=0$ into Eq. (6) and reduce Eqs. (6)-(8) to

$$
\frac{d}{d \tau}\left(\begin{array}{l}
G_{1 \xi} \\
G_{2 \xi}
\end{array}\right)=i\left(\begin{array}{cc}
\Delta_{1}+i \tilde{\kappa}_{1} / 2 & -i N \operatorname{Ig}_{1} g_{2} / \Gamma \\
-i N \operatorname{Ig}_{1} g_{2} / \Gamma & \Delta_{2}+i \tilde{\kappa}_{2} / 2
\end{array}\right)\left(\begin{array}{l}
G_{1 \xi} \\
G_{2 \xi}
\end{array}\right),
$$

which represents two identical $2 \times 2$ matrices and characterizes the coupling of the two cavity modes. The eigenvalues of the matrix above read

$$
\begin{aligned}
\lambda_{ \pm}= & \frac{\Delta_{1}+\Delta_{2}}{2}+i \frac{\tilde{\kappa}_{1}+\tilde{\kappa}_{2}}{4} \\
& \pm\left\{\left[\left(\Delta_{1}-\Delta_{2}\right) / 2+i\left(\tilde{\kappa}_{1}-\tilde{\kappa}_{2}\right) / 4\right]^{2}-\left(N I g_{1} g_{2} / \Gamma\right)^{2}\right\}^{1 / 2},
\end{aligned}
$$

where the notation $\tilde{\kappa}_{\xi}=\kappa_{\xi}-2 N I g_{\xi}^{2} / \Gamma$ reveals that increasing $N I g_{\xi}^{2} / \Gamma$ effectively reduces the linewidths. Inspection of Eq. (10) shows that the dependence of the eigenvalues on the involved parameters behaves quite differently from the case of conventional NMC, Eq. (1). For $\Delta_{1}=\Delta_{2}$ and $g_{1}=g_{2}$, the square-root term of $\lambda_{ \pm}$remains imaginary and modifies the peak widths independently of how large the effective coupling $\left|N I g_{1} g_{2} / \Gamma\right|$ is chosen in comparison with $\left|\kappa_{1}-\kappa_{2}\right|$. In the case when $\kappa_{1}=\kappa_{2}$ and $g_{1}=g_{2}$, the square-root term leads to 

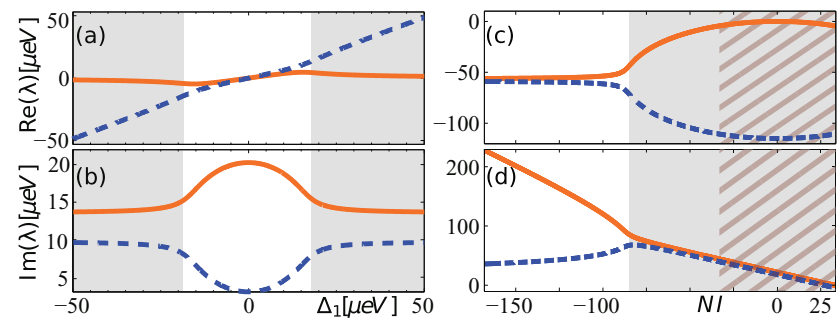

FIG. 1. (Color online) Peak positions (a),(c) and peak widths (b),(d) [cf. Eq. (10)] vs $\Delta_{1}$ for fixed pump strength [(a),(b), $I=$ $0.3]$ and vs $N I$ [(c),(d), $\left.\Delta_{1}=115 \mu \mathrm{eV}\right], N=42, \kappa_{1}=44 \mu \mathrm{eV}$, $\kappa_{2}=36 \mu \mathrm{eV}, \Delta_{2}=0, \Gamma=1.38 \mathrm{meV}, g_{1}=30.4 \mu \mathrm{eV}$, and $g_{2}=$ $30.3 \mu \mathrm{eV}$. The shaded regions indicate incoherent coupling. The hatched area indicates the range of $N I$ that corresponds to our experimental results presented below (see Fig. 3). The values of $\kappa_{\xi}$ and $\Delta_{\xi}$ are measured in the experiment, and the values of $N, \Gamma$, and $g_{\xi}$ are estimated in correspondence to the experiment.

two regimes. For $\left|N I g_{1} g_{2} / \Gamma\right|<\left|\Delta_{1}-\Delta_{2}\right| / 2$, the regime of incoherent coupling, the term is real and modifies the peak positions of the modes. On the other hand, in the regime of coherent coupling, i.e., for $\left|N I g_{1} g_{2} / \Gamma\right|>\left|\Delta_{1}-\Delta_{2}\right| / 2$, it becomes imaginary and modifies the peak widths of the modes. Note that this striking behavior is qualitatively opposite from that of conventional NMC. Furthermore, since the effective coupling $\left|N I g_{1} g_{2} / \Gamma\right|$ is proportional to $|I|$, the experimental observation of the coherent regime of collective coupling requires $|I|$ to be close to unity.

To demonstrate the unconventional NMC we plot the real and imaginary parts of the eigenvalues for a coupled bimodal cavity system in Fig. 1. The dependence on the detuning $\Delta_{1}\left(\Delta_{2}=0\right)$ [Figs. 1(a) and 1(d)] reveals that in the near-resonant region where the coherent coupling regime is maintained, a splitting of the imaginary parts of the eigenvalues (peak widths) is observed. For increasing detuning between the cavity modes, in the incoherent coupling regime, the eigenvalues demonstrate splitting in the real parts (peak positions) and locking of the peaks widths. To illustrate the dependence on the effective coupling rate the real and imaginary parts of the eigenvalues are presented as functions of $N I$ in Figs. 1(c) and 1(d). In the regime of incoherent coupling, for small effective coupling rate (small $N|I|$ ), the splitting of the peak positions is observed. In the regime of coherent coupling the splitting of the peak widths increases for an increasing effective coupling rate, whereas in the case of the conventional coherent NMC, Eq. (1), the splitting of the mode resonances increases for increasing $N$ [31].

We would like to note that despite formal similarities the collective NMC described by Eq. (9) [cf. Eqs. (6)-(8)] is fundamentally different from the nonlinear equations of motion for the field amplitudes of a bimodal laser obtained within the semiclassical theory [32]. First of all, the semiclassical theory neglects spontaneous emission and therefore describes neither the laser fluctuations nor the field coherence times. Most importantly, the mode coupling in the semiclassical theory controls only the existence and stability of the dualmode operation. But the frequency characteristics of the modes emerge from the self-consistency equations of wave equations-and not from the coupling matrix of the system.

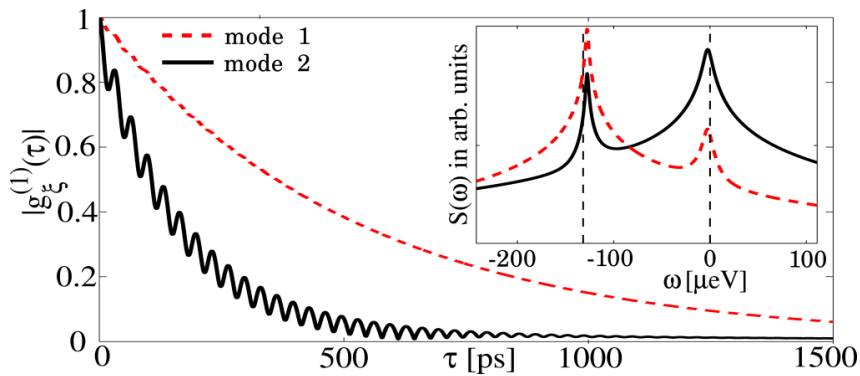

FIG. 2. (Color online) Absolute values of the autocorrelation functions and the frequency spectra (inset, semilogarithmic scale) for the emission in the basis of the cavity modes for $I=0.65$, $\Delta_{1}=115 \mu \mathrm{eV}$, and an estimated cavity-enhanced QD spontaneous emission rate of $20 \mathrm{~ns}^{-1}$. Other parameter values are from Figs. 1(c) and $1(\mathrm{~d})$. The vertical lines mark the passive cavity-mode frequencies.

Spectra. Importantly, the effects discussed above can be deduced starting with the more general Eqs. (6)-(8). In the following we use Eqs. (6)-(8) to obtain $\tau$-dependent expressions for the autocorrelation functions. Note that the initial values of the quantities $P_{\xi}(\tau)$ and $G_{\zeta \xi}(\tau)$ and the values of the $\tau$-time-independent $I(t)$ of the gain medium are taken as the stationary solutions of $t$-time-dependent problem [25]. Figure 2 reveals that in the regime of incoherent coupling the coherence times of the dominant and inferior modes, which correspond to the decay rates of $\left|g_{1}^{(1)}(\tau)\right|$ and $\left|g_{2}^{(1)}(\tau)\right|$, respectively, are of the same order of magnitude. This counterintuitive behavior, which has been experimentally observed earlier [see Fig. 2(b) of Ref. [25]], is particularly interesting considering that the inferior mode features large superthermal intensity fluctuations with $g^{(2)}(0) \approx 3$ [Fig. 2(f) of Ref. [25]]. The spectra of both modes, shown in the inset of Fig. 2, exhibit a two-peak structure according to the eigenvalues in Eq. (10). Indeed, for the chosen parameters $N I=27.3$, which correspond to the case of the incoherent coupling, Figs. 1(c) and 1(d) reveal the splitting of the peak positions and locking of the widths. Obviously, emission in the basis of the two cavity modes carries both "bare" frequencies of the passive cavity modes due to NMC via the common gain medium. The emission peak positions and widths are established by the real and imaginary parts of the eigenvalues $\lambda_{ \pm}$, since every mode carries both basis eigenvectors. The mode coupling is also associated with the oscillations of $\left|g_{\xi}^{(1)}(\tau)\right|$ [this is easy to see in Fig. 2 for $\left|g_{2}^{(1)}(\tau)\right|$ but holds true for $\left.\left|g_{1}^{(1)}(\tau)\right|\right]$. In particular, the oscillation amplitudes are attributed to the corresponding frequency spectra peak heights, whereas the oscillation frequency is defined by the peak position difference, which in turn is related to the relative detuning of the cavity modes. Importantly, the oscillations of $\left|g_{\xi}^{(1)}(\tau)\right|$ originate from mode coupling and need to be distinguished from those reported in Ref. [33], which arise from the interference of emission in two different polarization directions.

Experimental realization. Here we present the experimental investigation of NMC in bimodal lasers, where we study electrically pumped micropillar lasers based on a doped planar microcavity sample with a single layer of self-assembled InGaAs QDs acting as active medium (for technological details, see Refs. $[34,35])$. An asymmetry of the cross section 

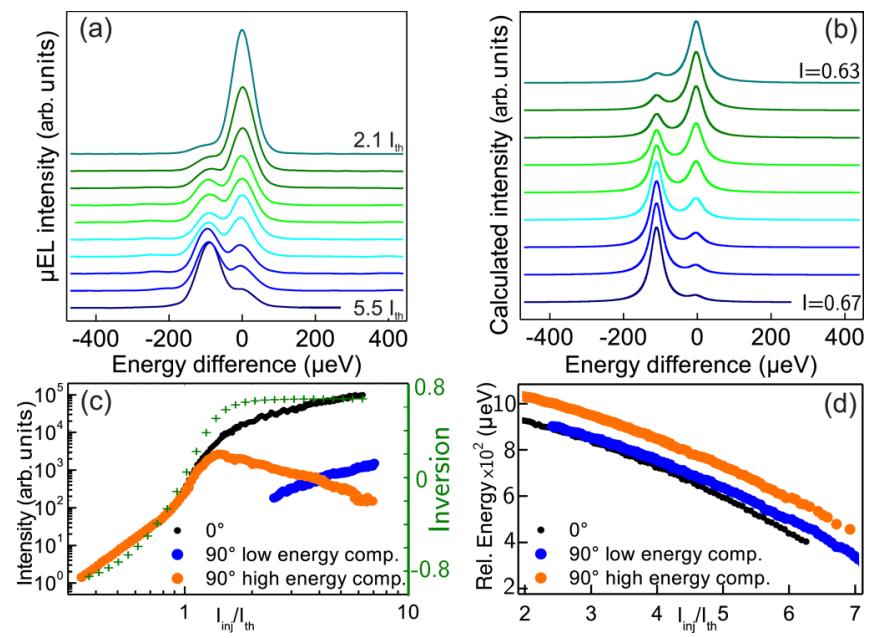

FIG. 3. (Color online) (a) Injection-current-dependent $\quad \mu \mathrm{EL}$ emission spectra in $90^{\circ}$ polarization for $2.1 \leqslant I_{\text {inj }} / I_{\text {th }} \leqslant 5.5$, plotted relative to the high-energy peak values to compensate for an injection-current-dependent shift of the emission energy [see (d)]. (b) Calculated frequency spectrum in $90^{\circ}$ polarization for inversion values $0.63 \leqslant I \leqslant 0.67$. Parameter values are the same as in Fig. 2 . (c) Calculated inversion (green, crosses) vs injection current and integrated $\mu \mathrm{EL}$ intensity for $0^{\circ}$ and $90^{\circ}$ polarizations. (d) Emission energy (relative to the reference point $1.366 \mathrm{eV}$ ) of the $0^{\circ}$ component and the two-mode features in $90^{\circ}$ detection vs injection current.

of the pillar and/or the upper ring-shaped electrical contact lifts the degeneracy of the resonator fundamental mode, and thus two frequency-separated linearly (orthogonally) polarized cavity modes are supported [36]. The micropillar laser under study has a diameter of $3.6 \mu \mathrm{m}$, and the two modes of $0^{\circ}$ and $90^{\circ}$ polarizations and $Q$ factors of 10000 and 10800 are split by $115 \mu \mathrm{eV}$. The emission has been investigated at low temperature $(10 \mathrm{~K})$ by a microelectroluminescence ( $\mu \mathrm{EL}$ ) setup (spectral resolution $20 \mu \mathrm{eV}$ ). A linear polarizer in combination with a $\lambda / 4$-wave plate is installed to perform polarization-resolved measurements.

The input-output dependence of the emission in detection angles of $0^{\circ}$ and $90^{\circ}$ is depicted in Fig. 3(c). The emission mode in $0^{\circ}$ polarization shows a threshold current of about $I_{t h}=4 \mu \mathrm{A}$. The smooth transition at threshold and the Sshaped input-output characteristics indicate the high- $\beta$ lasing with $\beta \approx 0.2$ [25]. A similar behavior is observed for emission in $90^{\circ}$ polarization up to $I_{\text {inj }} \cong 1.5 I_{\text {th }}$. At higher injection currents, saturation and even a decrease of the output intensity is observed. This anticorrelation between emission of the dominant mode in $0^{\circ}$ and the inferior mode in $90^{\circ}$ polarization is explained by means of the microscopic semiconductor model in terms of gain competition [25]. Moreover, the model allows us to determine the inversion $I$ vs pump rate which changes from -0.8 to 0.8 for the parameter values used [see Fig. 3(c)]. The corresponding NI range is indicated in Figs. 1(c) and 1(d) as the hatched area.

Interestingly, the intensity of emission in $90^{\circ}$ polarization increases again for $I_{\text {inj }} \gtrsim 4 I_{\text {th }}$ [see Fig. 3(c)]. To analyze this feature we study the emission spectra of the laser for different injection currents. While in the $0^{\circ}$ orientation emission a single peak is observed (not shown), for the $90^{\circ}$ orientation at injection currents exceeding about $1.5 I_{\text {th }}$ a transition of a single emission peak into a doublet occurs [see Fig. 3(a)], where the intensity of the low-energy component rises with increasing current and dominates for $I_{\text {inj }}>5 I_{\text {th }}$. This doublepeak feature of the $90^{\circ}$ orientation emission and its peculiar current dependence are in very good agreement with the calculated emission spectra presented in Fig. 3(b) for incoherent collective coupling [37]. Indeed, for the range of the chosen parameters $N I \approx 26-28$, which, according to Figs. 1(c) and $1(\mathrm{~d})$, corresponds to the region of incoherent coupling.

In Fig. 3(d) mode energies of $0^{\circ}$ and $90^{\circ}$ polarizations vs injection current are plotted. At low injection currents the single-peak emission in both polarization directions corresponds to the bare frequencies of the cavity modes. Moreover, the low-energy component of the $90^{\circ}$ emission for low injection currents coincides spectrally with the $0^{\circ}$ emission, but at high excitation currents it approaches the energy of the high-energy peak in the $90^{\circ}$ orientation. This clearly shows that this emission does not originate from possible cross-talk between the $0^{\circ}$ and $90^{\circ}$ components, but is in accordance with the theoretical prediction in Fig. 1(c), namely, that the peak positions approach each other with increasing $N I$.

In summary, we have demonstrated the existence of collective NMC in bimodal microlasers. In contrast to the conventional case, here, in the coherent coupling regime, the increase of the effective coupling rate produces a splitting of the linewidths instead of the frequencies. In the incoherent coupling regime, increasing effective coupling induces splitting of frequencies and locking of linewidths. The consequence is a double-peak structure of the output spectra of the modes and large coherence times for both dominant lasing and inferior modes, which we have confirmed experimentally in QD-based bimodal micropillar lasers. The latter offer unique possibilities to study collective coupling, since the stimulated emission of the dominant mode leads to clamping of the carrier density with large inversion $(I \approx 0.8)$, while the inferior mode experiences collective coupling mediated by multiple inverted emitters. Note that the unconventional NMC is not a semiconductor effect, but can be observed in the case of the collective weak interaction of two modes with a mesoscopic number of atoms. We expect to observe further interesting effects related to NMC in bimodal lasers in the coherent coupling regime which could be accessed by using micropillar cavities with small mode splitting and larger inversion rate and/or number of involved QDs.
[1] M. Brune, F. Schmidt-Kaler, A. Maali, J. Dreyer, E. Hagley, J. M. Raimond, and S. Haroche, Phys. Rev. Lett. 76, 1800 (1996).
[2] J. McKeever, J. R. Buck, A. D. Boozer, A. Kuzmich, H.-C. Nägerl, D. M. Stamper-Kurn, and H. J. Kimble, Phys. Rev. Lett. 90, 133602 (2003). 
[3] S. Nußmann, M. Hijlkema, B. Weber, F. Rohde, G. Rempe, and A. Kuhn, Phys. Rev. Lett. 95, 173602 (2005).

[4] J. P. Reithmaier, G. Sek, A. Löffler, C. Hofmann, S. Kuhn, S. Reitzenstein, L. V. Keldysh, V. D. Kulakovskii, T. L. Reinecke, and A. Forchel, Nature (London) 432, 197 (2004).

[5] T. Yoshie, A. Scherer, J. Hendrickson, G. Khitrova, H. M. Gibbs, G. Rupper, C. Ell, O. B. Shchekin, and D. G. Deppe, Nature (London) 432, 200 (2004).

[6] B. W. Shore and P. L. Knight, J. Mod. Opt. 40, 1195 (1993).

[7] C. Weisbuch, M. Nishioka, A. Ishikawa, and Y. Arakawa, Phys. Rev. Lett. 69, 3314 (1992).

[8] T. J. Kippenberg and K. J. Vahala, Science 321, 1172 (2008).

[9] J. M. Dobrindt, I. Wilson-Rae, and T. J. Kippenberg, Phys. Rev. Lett. 101, 263602 (2008).

[10] C. Monroe, Nature (London) 416, 238 (2002).

[11] M. Tavis and F. W. Cummings, Phys. Rev. 170, 379 (1968).

[12] L. C. Andreani, G. Panzarini, and J.-M. Gérard, Phys. Rev. B 60, 13276 (1999).

[13] S. Reitzenstein, A. Löffler, C. Hofmann, A. Kubanek, M. Kamp, J. P. Reithmaier, A. Forchel, V. D. Kulakivskii, L. V. Keldysh, I. V. Ponomarev, and T. L. Reinecke, Opt. Lett, 31, 1738 (2006).

[14] F. Albert, K. Sivalertporn, J. Kasprzak, M. Strauß, C. Schneider, S. Höfling, M. Kamp, A. Forchel, S. Reitzenstein, E. Muljarov, and W. Langbein, Nat. Commun. 4, 1747 (2013).

[15] M. G. Raizen, R. J. Thompson, R. J. Brecha, H. J. Kimble, and H. J. Carmichael, Phys. Rev. Lett. 63, 240 (1989).

[16] A. K. Tuchman, R. Long, G. Vrijsen, J. Boudet, J. Lee, and M. A. Kasevich, Phys. Rev. A 74, 053821 (2006).

[17] A. Wickenbrock, M. Hemmerling, G. R. M. Robb, C. Emary, and F. Renzoni, Phys. Rev. A 87, 043817 (2013).

[18] G. Khitrova, H. M. Gibbs, F. Jahnke, M. Kira, and S. W. Koch, Rev. Mod. Phys. 71, 1591 (1999).

[19] P. Meystre and M. Sargent III, Elements of Quantum Optics, 2nd ed. (Springer-Verlag, Berlin, 1991).

[20] A. Siegman, Lasers (University Science Books, Mill Valley, CA, 1986).

[21] L. W. Hillman, J. Krasiński, R. W. Boyd, and C. R. Stroud, Phys. Rev. Lett. 52, 1605 (1984).
[22] C. Y. Wang, L. Diehl, A. Gordon, C. Jirauschek, F. X. Kärtner, A. Belyanin, D. Bour, S. Corzine, G. Höfler, M. Troccoli, J. Faist, and F. Capasso, Phys. Rev. A 75, 031802 (2007).

[23] B. Dayan, A. S. Parkins, T. Aoki, E. P. Ostby, K. J. Vahala, and H. J. Kimble, Science 319, 1062 (2008).

[24] A. Majumdar, M. Bajcsy, A. Rundquist, and J. Vučković, Phys. Rev. Lett. 108, 183601 (2012).

[25] H. A. M. Leymann, C. Hopfmann, F. Albert, A. Foerster, M. Khanbekyan, C. Schneider, S. Höfling, A. Forchel, M. Kamp, J. Wiersig, and S. Reitzenstein, Phys. Rev. A 87, 053819 (2013).

[26] M. Lax, Phys. Rev. 157, 213 (1967).

[27] J. Wiersig, Phys. Rev. B 82, 155320 (2010).

[28] H. A. M. Leymann, A. Foerster, and J. Wiersig, Phys. Rev. B 89, 085308 (2014).

[29] G. W. Gardiner and P. Zoller, Quantum Noise, 3rd ed. (SpringerVerlag, Berlin, 2001).

[30] Z. I. Alferov, Rev. Mod. Phys. 73, 767 (2001).

[31] R. J. Thompson, G. Rempe, and H. J. Kimble, Phys. Rev. Lett. 68, 1132 (1992).

[32] M. Sargent III, M. O. Scully, and W. E. Lamb, Laser Physics (Addison-Wesley, Reading, MA, 1974).

[33] S. Ates, S. M. Ulrich, P. Michler, S. Reitzenstein, A. Löffler, and A. Forchel, Appl. Phys. Lett. 90, 161111 (2007).

[34] S. Reitzenstein, T. Heindel, C. Kistner, F. Albert, T. Braun, C. Hopfmann, P. Mrowinski, M. Lermer, C. Schneider, S. Höfling, M. Kamp, and A. Forchel, IEEE J. Sel. Top. Quantum Electron. 17, 1670 (2011).

[35] F. Albert, C. Hopfmann, S. Reitzenstein, C. Schneider, S. Höfling, L. Worschech, M. Kamp, W. Kinzel, A. Forchel, and I. Kanter, Nat. Commun. 2, 366 (2011).

[36] S. Reitzenstein, C. Hofmann, A. Gorbunov, M. Straub, S. H. Kwon, C. Schneider, A. Löffler, S. Höfling, M. Kamp, and A. Forchel, Appl. Phys. Lett. 90, 251109 (2007).

[37] These spectra were convoluted with a Lorentzian to take into account the spectral resolution of $20 \mu \mathrm{eV}$ of the experimental setup. 